\title{
Clinical Analysis of Benign and Malignant Nasal Septal Tumors
}

\author{
Dong Hoon Lee ${ }^{D}$, Sang Chul Lim ${ }^{(D)}$, Sung Ho Yoon, Tae Gu Kang, and Jong Min Park \\ Department of Otolaryngology-Head and Neck Surgery, Chonnam National University Medical School \& \\ Chonnam National University Hwasun Hospital, Hwasun, Korea
}

\section{양성 및 악성 비중격 종양의 임상적 분석}

이동훈 · 임상철 · 윤성호 · 강태구 · 박종민

전남대학교 의과대학 화순전남대학교병원 이비인후과학교실

\author{
Received July 31,2018 \\ Revised October 5, 2018 \\ Accepted October 29, 2018 \\ Address for correspondence \\ Sang Chul Lim, MD, PhD \\ Department of Otolaryngology- \\ Head and Neck Surgery, \\ Chonnam National University \\ Medical School \& Chonnam \\ National University Hwasun \\ Hospital, 322 Seoyang-ro, Hwasun \\ 58128 , Korea \\ Tel $+82-61-379-8190$ \\ Fax +82-61-379-7761 \\ E-mail limsc@chonnam.ac.kr
}

Background and Objectives The purpose of this study was to review the clinical characteristics and treatment outcomes of benign and malignant nasal septal tumors.

Subjects and Methods A total of 34 patients who underwent the treatment for nasal septal tumor between 2004 and 2015 were included in this study. Demographic characteristics, sinonasal chief complaints, size and localization of nasal septal tumor, imaging findings, surgical method, histopathologic results, treatment outcomes, and postoperative complications were reviewed.

Results Of the 34 patients with nasal septal tumor used in this study, 22 (64.7\%) had benign tumors and 12 (35.3\%) had malignant tumors. The most common sinonasal complaint of both benign and malignant nasal septal tumors was nasal obstruction. The most common benign tumor was inverted papilloma $(\mathrm{n}=7)$, whereas the most common malignant tumors were malignant melanoma $(n=2)$, plasmacytoma $(n=2)$, and metastatic carcinoma $(n=2)$. All patients except two cases underwent endoscopic tumor removal. There were 4 recurrences in malignant nasal septal tumors. There was no recurrence in benign tumors. No major complications resulting from surgical intervention were found.

Conclusion Endoscopic surgery with complete excision is a safe and effective procedure for benign nasal septal tumors. Recurrence is more common in malignant nasal septal tumor. Therefore, long term follow-ups with regular radiologic and endoscopic examinations are necessary for patients with malignant nasal septal tumor. Korean J Otorhinolaryngol-Head Neck Surg 2019;62(4):228-32

Key Words Neoplasms · Transnasal endoscopic surgery · Nasal septum · Radiotherapy.

\section{Introduction}

Nasal septal tumors (benign or malignant) are rare diseases. The incidence of malignant nasal septal tumors is $2.7-8.4 \%$ of nasal and paranasal malignancies. ${ }^{1-5)}$ The symptoms of nasal septal tumors are usually nonspecific, such as nasal ob-

This is an Open Access article distributed under the terms of the Creative Commons Attribution Non-Commercial License (https://creativecommons.org/licenses/by-nc/4.0) which permits unrestricted non-commercial use, distribution, and reproduction in any medium, provided the original work is properly cited. struction or nasal bleeding. ${ }^{1-3,5)}$ The differential diagnosis of nasal septal tumors depends on various pathologies, ranging from benign tumors to malignant tumors. ${ }^{1-6)}$ Because of their rarity, discrepancies among clinical and histologic aspects of nasal septal tumors have occurred. ${ }^{2,3)}$ In addition, the precise treatment for nasal septal tumor remains unlcear. ${ }^{5)}$ Therefore, the objective of this study was to review the clinical characteristics and treatment outcomes of nasal septal tumors based on our 12-year experience. 


\section{Subjects and Methods}

After obtaining approval from the Institutional Review Board of Chonnam National University Hwasun Hospital, this study was performed (IRB No. CNUHH-2018-119). A total of 34 patients who underwent treatment for nasal septal tumor between 2004 and 2015 were included in this study. This study population was subdivided into benign $(\mathrm{n}=22)$ and malignant tumor $(\mathrm{n}=12)$.

Their demographic characteristics, sinonasal chief complaints, size and localization of nasal septal tumor, imaging findings, surgical method, histopathologic results, treatment outcomes, and postoperative complications were reviewed.

All patients underwent endoscopic examination and radiologic scans before the operation to assess the extent of the lesion and aid treatment planning. All patients except two cases underwent endoscopic tumor removal. One patient underwent radiofrequency ablation due to poor general condition at outpatient department. Another patient was only performed endoscopic biopsy. Among the 12 patients with malignant nasal septal tumors, five underwent postoperative radiotherapy. All cases of nasal septal tumors were confirmed histopathologically.

Fisher's exact test was used to determine the associations between two categorical variables. SPSS version 14.0. (SPSS Inc., Chicago, IL, USA) was used for all statistical analyses. Statistical significance was defined at $p$-value $<0.05$.

\section{Results}

Of the 34 patients with nasal septal tumors, 22 (64.7\%) had benign tumors and $12(35.3 \%)$ had malignant tumors. Clinical findings in both benign and malignant nasal septal tumors are summarized in Table 1.

Of the 22 patients with benign nasal septal tumor, 16 were males and 6 were females. Their mean age was $36.0 \pm 16.4$ years (range, 10-72 years). Sinonasal complaints included nasal obstruction (8 patients, 36.4\%) and nasal bleeding (7 patients, $31.8 \%$ ). The rest patients had incidental diagnosis (7 patients, 31.8\%). Of the 22 benign lesions, 15 (68.2\%) were located in the cartilage septum and $7(31.8 \%)$ were located in the bony septum. The mean tumor size was $1.7 \pm 0.8 \mathrm{~cm}$ (range, $0.5-3 \mathrm{~cm}$ ). Endoscopic surgery was performed for all patients with benign nasal septal tumor. The tumor and surrounding normal tissue were removed with a safety margin of about $5 \mathrm{~mm}$, and some cartilage or bone involvement were removed. Two of 22 patients underwent postoperative reconstruction, 1 with skin graft, and 1 with inferior turbinate flap. According to histopathologic results, the most common one $(n=7)$ was inverted papilloma (Fig. 1), followed by hemangioma $(n=5)$, nasal polyp $(n=3)$, squamous papilloma $(n=3)$, pleomorphic adenoma $(n=2)$, and pyogenic granuloma $(n=2)$. Their mean follow-up period after surgery was $93.1 \pm 36.3$ months (range, 14-153 months). There was no recurrence in benign nasal septal tumor.

Of the 12 patients with malignant nasal septal tumor, 8 were males and 4 were females. Their mean age was $57.0 \pm 10.0$ years (range, 21-75 years). The most common sinonasal complaints were nasal obstruction (7 patients, 58.3\%), followed by nasal bleeding (4 patients, 33.3\%), and nasal discharge (1 patient, 8.3\%). Of the 12 lesions, 4 (33.3\%) were located in the cartilage septum and $8(66.7 \%)$ were located in the bony sep-

Table 1. Clinical findings of patients with benign or malignant nasal septal tumors

\begin{tabular}{lcc}
\hline \multicolumn{1}{c}{ Variables } & Benign tumors $(\mathrm{n}=22)$ & Malignant tumors $(\mathrm{n}=12)$ \\
\hline Age (years) & $10-72(36.0 \pm 16.4)$ & $21-75(57.0 \pm 10.0)$ \\
Sex $(\mathrm{M}: \mathrm{F})$ & $16: 6$ & $8: 4$ \\
Chief sinonasal complaint & Nasal obstruction $(36.4 \%)$ & Nasal obstruction $(58.3 \%)$ \\
Duration of complaint (months) & $0.3-240(34.2 \pm 70.7)$ & $0.5-6(2.0 \pm 1.5)$ \\
Nasal cavity (R:L) & $11: 11$ & $5: 7$ \\
Location (cartilage:bone) & $15: 7$ & $4: 8$ \\
Tumor size (cm) & $0.5-3(1.7 \pm 0.8)$ & $0.5-4.2(2.5 \pm 1.1)$ \\
Surgery & Endoscopic excision (100\%) & Endoscopic excision $(83.3 \%)$ \\
Histopathologic result (m/c) & Inverted papilloma (31.8\%) & Plasmacytoma (16.7\%) \\
& - & Metastatic carcinoma $(16.7 \%)$ \\
Follow-up (months) & - & $5-126(53.9 \pm 39.4)$ \\
Recurrence & $14-153(93.1 \pm 36.3)$ & $4(33.3 \%)$
\end{tabular}

M: male, F: female, R: right, L: left, m/c: most common 

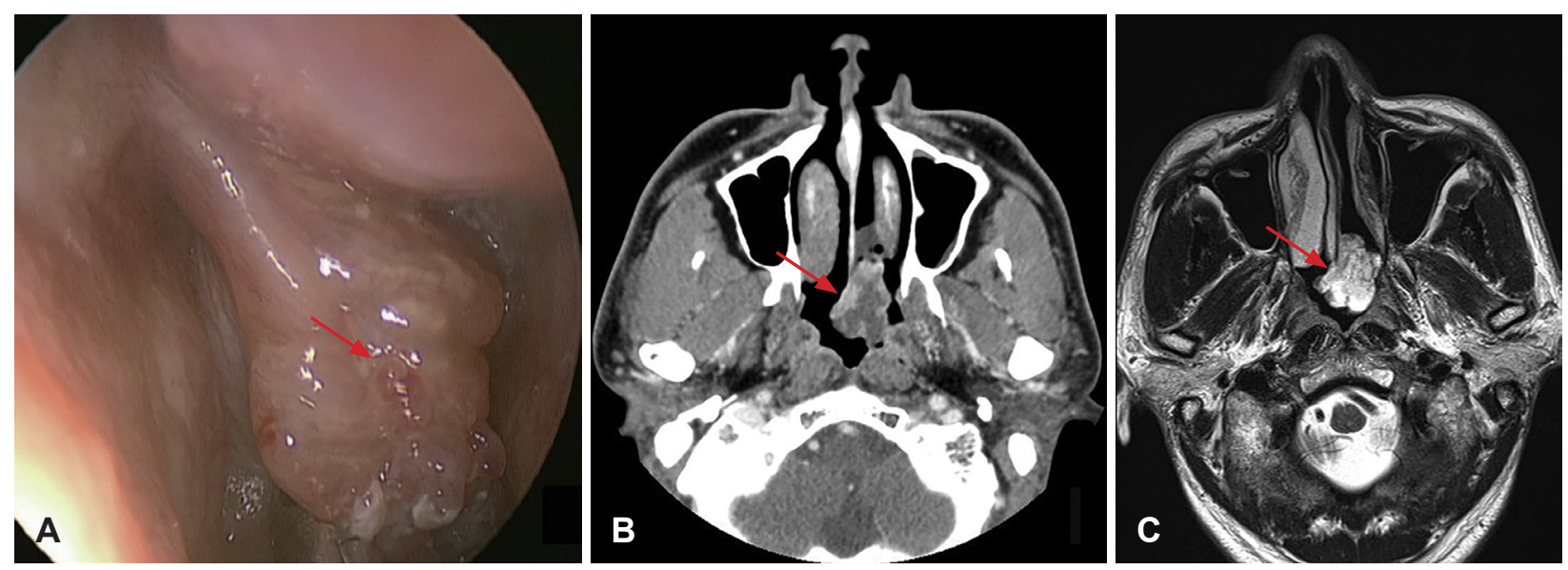

Fig. 1. Nasal endoscopy (A), CT scan (B), and T2-weighted image MRI scan (C) in a 47-year-old male patient showing a pedunculated inverted papilloma originating from posterior nasal septum and extending to the nasopharynx.

tum. The mean tumor size was $2.5 \pm 1.1 \mathrm{~cm}$ (range, $0.5-4.2$ $\mathrm{cm})$. Endoscopic surgery for malignant nasal septal tumor was performed in 10 patients $(83.3 \%)$. In the case of malignant tumors, we tried to secure a safety margin of about $1 \mathrm{~cm}$ and removed cartilage or bone tissue. Frozen biopsy was performed to determine whether additional resection was necessary. One patient underwent postoperative reconstruction with skin graft. One patient with nasal septal metastatic hepatocellular cell carcinoma (HCC) underwent radiofrequency ablation, because the poor general condition due to systemic metastasis of HCC. Another patient with nasal septal squamous cell carcinoma was only performed endoscopic biopsy. This patient underwent radiotherapy immediately after the diagnosis instead of the operation. Histopathologic results included malignant melanoma $(n=2)$, sinonasal extramedullary plasmacytoma $(\mathrm{n}=2)$, metastatic carcinoma $(\mathrm{HCC}$, esophageal cancer, $n=2)$, chondrosarcoma $(n=1)$, hemangiopericytoma $(n=1)$, leiomyosarcoma $(n=1)$, mucoepidermoid carcinoma $(n=1)$, adenoid cystic carcinoma $(n=1)$, and squamous cell carcinoma $(n=1)$. Their mean follow-up period after surgery was 53.9 \pm 39.4 months (range, 5-126 months). There were four recurrence of malignant nasal septal tumor. All patients with malignant nasal septal tumor except two were alive at the time of the last follow-up.

Recurrence of malignant nasal septal tumor was identified in $4(33.3 \%)$ patients, including two patients with metastatic carcinoma and two patients with plasmacytoma. Two patients diagnosed as metastatic carcinoma developed multiple metastasis and died at 5 (patient with HCC) and 31 months (patient with esophageal cancer) after surgery, respectively. Of the two patients with plasmacytoma, one patient had re- currence 52 months later after the 1st endoscopic surgery, the other patient (Fig. 2) had three times of recurrence after two endoscopic surgeries. This patient had been disease-free after radiotherapy. There were no major complications resulting from surgical intervention.

\section{Discussion}

This study presented our 12-year experience of nasal septal tumors. This study showed that both benign and malignant nasal septal tumors were more common in males than in females (male-female ratio, 2.4:1), consistent with previous reports. $^{2,5)}$

The most common sinonasal complaints of nasal septal tumor is nasal obstruction. ${ }^{1-3,5)}$ The presenting symptoms are usually nonspecific, including nasal obstruction and nasal bleeding. ${ }^{1-3,5)}$ There was no statistical difference in nasal symptoms between benign and malignant nasal septal tumors. Some patients may delay seeking medical care for up to 240 months. More advanced diseases show nasal swelling, epiphora, diplopia, proptosis, or palatal ulceration. ${ }^{5,6)}$ In some patients with benign nasal septal tumors, early detection of disease without specific symptoms may be possible by endoscopic examination in local clinics.

Fifteen $(68.2 \%)$ benign nasal septal tumors were located in the cartilage septum while $8(66.7 \%)$ malignant nasal septal tumors were located in the bony septum. Benign Nasal septal tumor had the tendency ( $p=0.075)$ to occur in cartilage septum. There was no difference in the incidence of left and right nasal cavities between benign and malignant tumors. In this study, the tumor sizes of benign and malignant 

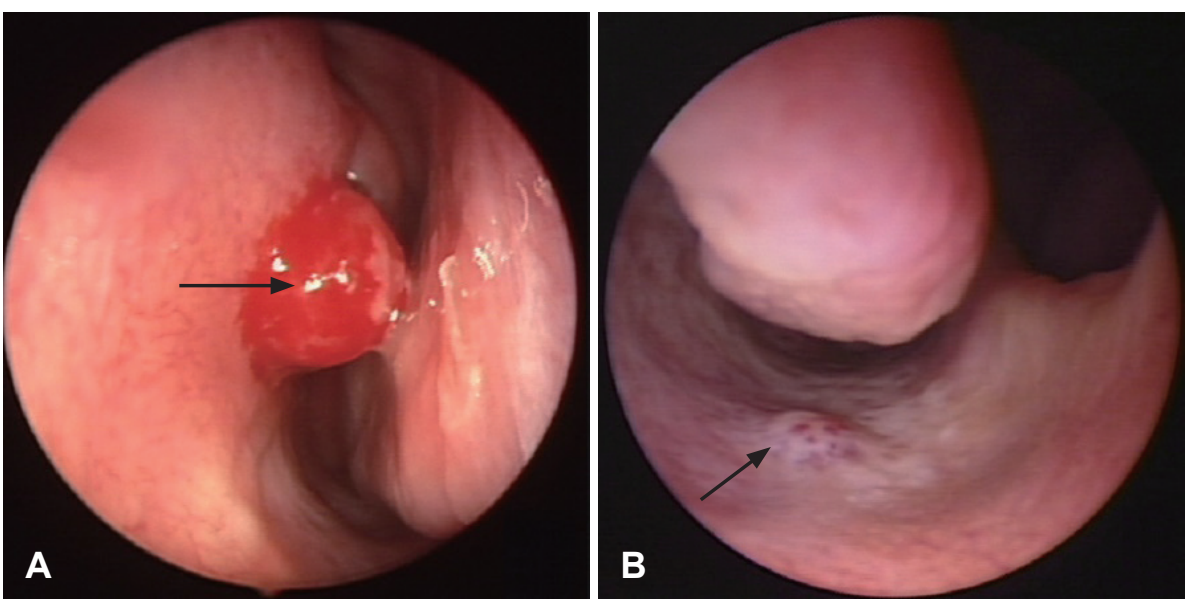

Fig. 2. Nasal endoscopy (A) in a 56-year-old male patient showing an easily bleeding plasmacytoma in the left nasal septum. The 1st recurrence of plasmacytoma in right nasal floor at 4 months after the 1st endoscopic surgery (B). The 2nd recurrence of plasmacytoma in posterior nasal septum at 1 month after the 2 nd endoscopic surgery (C). At the 42-months follow-up, nasal endoscopy shows no recurrence of plasmacytoma after radiotherapy (D).
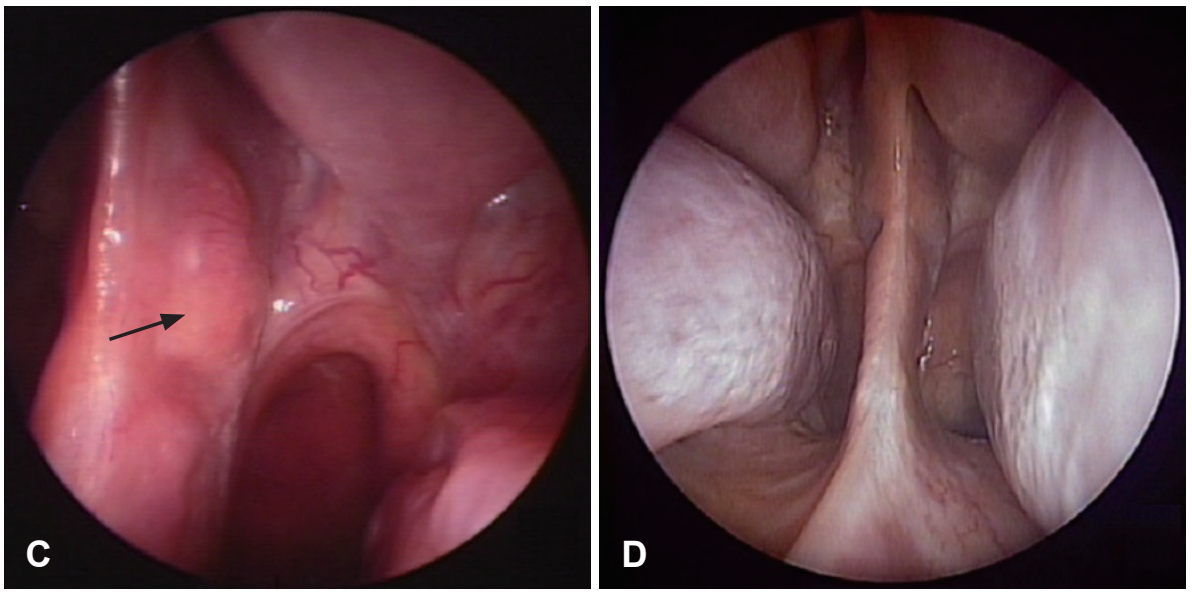

nasal septal tumors were $1.7 \pm 0.8 \mathrm{~cm}$ and $2.5 \pm 1.1 \mathrm{~cm}$, respectively. Malignant nasal septal tumor was found to be significantly $(p<0.05)$ bigger in size than benign nasal septal tumor.

Nasal endoscopy and radiologic examinations are excellent tools for diagnosing nasal septal tumors. ${ }^{6-8)}$ Nasal endoscopy is essential to make a tentative diagnosis for further investigations and for planning the surgical method. ${ }^{7)}$ Imaging examinations such as CT and MRI are useful for evaluating the extent and the characteristics of the tumor. They can be used to rule out anatomical variations and perform appropriate surgical planning. ${ }^{6-8)}$ We performed both endoscopic examination and radiologic scans in nasal septal tumors.

In this study, the most common benign tumor was inverted papilloma $(n=7)$. The most common malignant tumors were malignant melanoma, plasmacytoma, and metastatic carcinoma ( $\mathrm{n}=2$ for all). Differential diagnosis of nasal septal tumors depends on various pathologies such as squamous cell carcinoma, malignant melanoma, adenoid cystic carcinoma, adenocarcinoma, chondroma, Chondrosarcoma, osteosarcoma, schwannoma, lymphoma, and mucoepidermoid carcino- ma. ${ }^{1)}$ A tissue biopsy is essential for definite diagnosis. ${ }^{1,2,6)}$

Surgical removal is the generally accepted treatment of choice for benign nasal septal tumor. ${ }^{5,78)}$ Treatments for malignant nasal septal tumors include surgical therapy, radiation therapy, and combined therapies. ${ }^{1-6,8-11)}$ Various surgical procedures have been described, including Weber-Ferguson approach, sublabial transnasal approach, lateral rhinotomy approach, anterior craniofacial resection, and Lefort I osteotomy approach. ${ }^{1,9)}$ The surgical procedure depends on the size and location of the tumor. ${ }^{1,5,9)}$ Nasal septum is easily accessible. Endoscopic approach can remove small and localized nasal septal tumor., ${ }^{1,2,9)}$ Endoscopic surgery provides an excellent result in tumor control with obvious cosmetic benefits, shorter recovery time, and fewer side effects ${ }^{1,2,6,9)}$ as shown in our study This technique consists of complete removal of the nasal septal tumor with clear margin. For malignant nasal septal tumor, wide excision with $1 \mathrm{~cm}$ margins and the removal of full thickness mucosa, cartilage and/or bone is recommended. ${ }^{5)}$ In some malignant tumors, additional radiotherapy might be required to treat residual microscopic disease to achieve more 


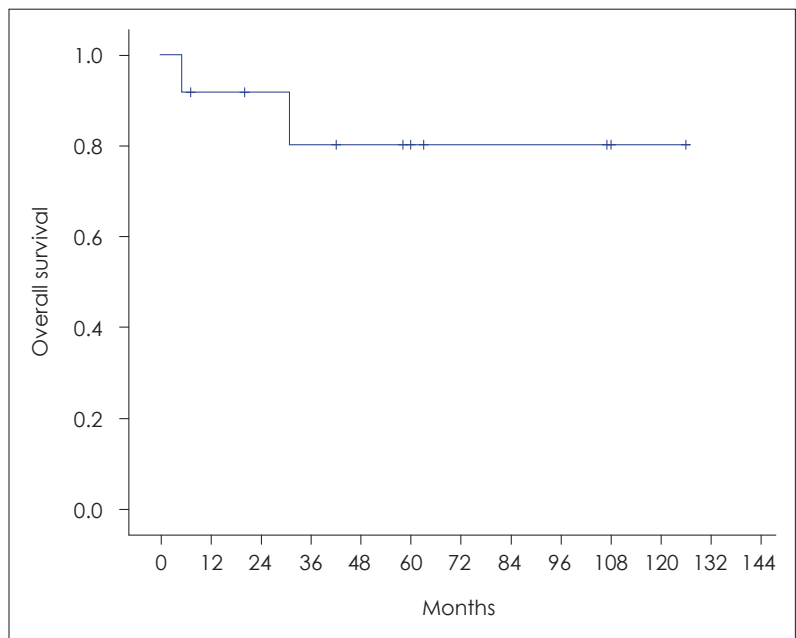

Fig. 3. Kaplan-Meier survival graphs for a series of 12 patients with malignant nasal septal tumors.

satisfactory local control and reduce the rate of recurrence..$^{2-4,10)}$ In this study, five patients among 12 patients with malignant nasal septal tumors underwent postoperative radiotherapy.

Recurrence is more $(p<0.05)$ common in malignant nasal septal tumors compared to that in benign ones. There were 4 recurrences (33.3\%) among malignant nasal septal tumors but none in benign tumors. Ho, et al. ${ }^{2}$ have reported a high local recurrence rate $(41.1 \%)$ in the early period of malignant nasal septal tumors. Among four recurrent patients, three patients had recurrence of the disease within 4 months. ${ }^{2)}$ However, one patient had the recurrence 52 months later after the 1st endoscopic surgery. Therefore, frequent and prolonged follow-up is mandatory for patients with malignant nasal septal tumors via nasal endoscopy.

The overall survival of patients with malignant nasal septal tumors is good compared with other cancers. ${ }^{2,4)}$ In this study, 3-year overall survival rate of malignant nasal septal tumor was $80.0 \%$ (Fig. 3).

In conclusion, Endoscopic surgery with complete excision is a safe and effective procedure for benign nasal septal tumors. Endoscopic resection for malignant nasal septal tumors may be possible due to the development of surgical instruments and techniques. Recurrence is more common in malignant nasal septal tumor. Therefore, long term followup with regular radiologic and endoscopic examinations are necessary for patients with malignant nasal septal tumor.

\section{ORCIDs}

Sang Chul Lim

https://orcid.org/0000-0001-5610-1554

Dong Hoon Lee

\section{REFERENCES}

1) Tai SY, Chien CY, Tai CF, Kuo WR, Huang WT, Wang LF. Nasal septum adenoid cystic carcinoma: A case report. Kaohsiung J Med Sci 2007;23(8):426-30.

2) Ho YM, Coman WB. Nasal septum malignancy. ANZ J Surg 2011; 81(7-8):533-6.

3) Akiyama K, Karaki M, Hosikawa H, Mori N. A massive adenoid cystic carcinoma of nasal septum progressed into the skull base. Auris Nasus Larynx 2013;40(2):239-42.

4) Beatty CW, Pearson BW, Kern EB. Carcinoma of the nasal septum: experience with 85 cases. Otolaryngol Head Neck Surg 1982;90(1): $90-4$.

5) DiLeo MD, Miller RH, Rice JC, Butcher RB. Nasal septal squamous cell carcinoma: A chart review and meta-analysis. Laryngoscope 1996;106(10):1218-22.

6) Magnano M, Boffano P, Machetta G, Garibaldi E, Delmastro E, Gabriele P. Chondrosarcoma of the nasal septum. Eur Arch Otorhinolaryngol 2015;272(3):765-72.

7) Salaria N, Sharma N, Garg U, Saluja SK, Agarwal R. Inflammatory septal nasal polyp. Iran J Otorhiolaryngol 2015;27(81):319-23.

8) Bizakis J, Nikolidakis A, Panayiotides J, Chimona T, Kyrmizakis D, Helidonis E. Vascular tumours of the nasal septum. J Otolaryngol 2002;31(3):170-2.

9) Coppit GL, Eusterman VD, Bartels J, Downey TJ. Endoscopic resection of chondrosarcoma of the nasal septum: A report of 2 cases. Otolaryngol Head Neck Surg 2002;127(6):569-71.

10) Sivaji N, Basavaraj S, Stewart W, Dempster J. Adenoid cystic carcinoma of the nasal septum. Rhinology 2003;41(4):253-4.

11) Lazzeri D, Agostini T, Giacomina A, Giannotti G, Colizzi L, De Rosa $\mathrm{M}$, et al. Malignant melanoma of the nasal septum. J Craniofac Surg 2010;21(6):1957-60 